\title{
Correction: Using financial incentives to promote physical activity in American Indian adolescents: A randomized controlled trial
}

\section{Kevin R. Short, Jennifer Q. Chadwick, Tamela K. Cannady, Dannielle E. Branam, David} F. Wharton, Mary A. Tullier, David M. Thompson, Kenneth C. Copeland

There is an error in the footnote of Table 1. "Increase from baseline to end of Phase 1 within group, $\mathrm{p}<0.03$ " should be corrected to "Increase from baseline to end of Phase 1 within group, $\mathrm{p}<0.05$ ". Please see the correct Table 1 here.

\section{f OPEn ACCESS}

Citation: Short KR, Chadwick JQ, Cannady TK, Branam DE, Wharton DF, Tullier MA, et al. (2020) Correction: Using financial incentives to promote physical activity in American Indian adolescents: A randomized controlled trial. PLOS ONE 15(3): e0231075. https://doi.org/10.1371/journal. pone. 0231075

Published: March 25, 2020

Copyright: $\odot 2020$ Short et al. This is an open access article distributed under the terms of the Creative Commons Attribution License, which permits unrestricted use, distribution, and reproduction in any medium, provided the original author and source are credited. 
Table 1. Clinical and physiological characteristics at baseline, changes at the end of Phase 1, and exercise performance in Phase 1.

\begin{tabular}{|c|c|c|c|c|}
\hline & \multicolumn{2}{|c|}{ Standard Group } & \multicolumn{2}{|c|}{ Incentive Group } \\
\hline & Baseline & Change at End of Phase & Baseline & Change at End of Phase \\
\hline $\mathrm{BMI}, \mathrm{kg} / \mathrm{m}^{2 *}$ & $34.9 \pm 8.5$ & $0.7 \pm 1.1 \dagger$ & $34.7 \pm 6.2$ & $0.7 \pm 1.8 \dagger$ \\
\hline BMI, percentile & $98 \pm 3$ & $0 \pm 1$ & $98 \pm 3$ & $0 \pm 1$ \\
\hline Body fat, $\%$ & $43.6 \pm 8.1$ & $0.9 \pm 2.7$ & $43.1 \pm 6.9$ & $0.1 \pm 4.2$ \\
\hline Fat-free mass, kg & $48.6 \pm 10.2$ & $0.6 \pm 2.9$ & $52.2 \pm 10.2$ & $0.9 \pm 4.3$ \\
\hline Waist circumference, $\mathrm{cm}$ & $104 \pm 16$ & $-1 \pm 5$ & $108 \pm 12$ & $0 \pm 8$ \\
\hline $\mathrm{VO}_{2}$ peak, $\mathrm{ml} / \mathrm{kg} \mathrm{FFM} / \mathrm{min}^{*}$ & $34.2 \pm 7.1$ & $3.4 \pm 5.7 \dagger$ & $35.2 \pm 8.7$ & $3.7 \pm 7.5 \dagger$ \\
\hline Steps per day & $6,404 \pm 3,425$ & $-869 \pm 2,318$ & $6,218 \pm 2,419$ & $483 \pm 3,401$ \\
\hline Glucose, $\mathrm{mmol} / \mathrm{l}$ & $5.3 \pm 0.5$ & $0.2 \pm 1.0$ & $5.1 \pm 0.4$ & $0.4 \pm 2.1$ \\
\hline Insulin, pmol/1 & $164.7 \pm 229.3$ & $-2.9 \pm 166.9$ & $119.7 \pm 94.3$ & $-11.4 \pm 80.3$ \\
\hline iHOMA2 (\%S) & $74.9 \pm 48.5$ & $-13.8 \pm 40.7$ & $68.6 \pm 44.2$ & $-4.9 \pm 26.2$ \\
\hline HbAlc (\%) & $5.4 \pm 0.3$ & $0.1 \pm 0.2 \dagger$ & $5.3 \pm 0.2$ & $0.1 \pm 0.6$ \\
\hline Exercise sessions performed & - & $26 \pm 16$ & - & $28 \pm 15$ \\
\hline Total MVPA time, $\mathrm{h}$ & - & $15.2 \pm 10.1$ & - & $15.0 \pm 8.1$ \\
\hline MVPA time per exercise session, minutes & - & $35 \pm 7$ & - & $32 \pm 8$ \\
\hline MVPA time in moderate intensity range, $\%$ & - & $63 \pm 18$ & - & $64 \pm 18$ \\
\hline Payments for exercise, USD\$ & - & $99 \pm 58$ & - & $245 \pm 144 \S$ \\
\hline
\end{tabular}

Values shown as mean $\pm \mathrm{SD}$. BMI, body mass index; $\mathrm{VO}_{2}$ peak, peak oxygen uptake during aerobic fitness test; FFM, fat-free mass; iHOMA2, integrated homeostatic model of assessment; HbAlc, hemoglobin Alc; MVPA, moderate-to-vigorous activity. The Standard group had 18 girls, 17 boys; mean age $14.2 \pm 2.4$ years at baseline. The Incentive group had 25 girls, 17 boys; mean age $14.4 \pm 2.3$ years at baseline.

* Main effect of time for entire cohort, $\mathrm{p}<0.01$.

$\dagger$ Increase from baseline to end of Phase 1 within group, $\mathrm{p}<0.05$.

$\S$ Difference between groups, $\mathrm{p}<0.01$.

https://doi.org/10.1371/journal.pone.0231075.t001

\section{Reference}

1. Short KR, Chadwick JQ, Cannady TK, Branam DE, Wharton DF, Tullier MA, et al. (2018) Using financial incentives to promote physical activity in American Indian adolescents: A randomized controlled trial. PLoS ONE 13(6): e0198390. https://doi.org/10.1371/journal.pone.0198390 PMID: 29856832 